\title{
Lindelöf Representations and (Non-)Holonomic Sequences
}

\author{
Philippe Flajolet \\ Algorithms Project, \\ INRIA Rocquencourt, F-78153 Le Chesnay (France) \\ Philippe.Flajolet AT inria.fr \\ Stefan Gerhold* \\ TU Vienna (Austria) and Microsoft Research-INRIA, Orsay (France) \\ sgerhold AT fam.tuwien.ac.at \\ Bruno Salvy \\ Algorithms Project, \\ INRIA Rocquencourt, F-78153 Le Chesnay (France) \\ Bruno.Salvy AT inria.fr
}

Submitted: Jun 9, 2009; Accepted: Dec 9, 2009; Published: Jan 5, 2010

Mathematics Subject Classifications: 11B83, 30E20, 33E20

Keywords: Holonomic sequence, $D$-finite function, Lindelöf representation

\begin{abstract}
Various sequences that possess explicit analytic expressions can be analysed asymptotically through integral representations due to Lindelöf, which belong to an attractive but somewhat neglected chapter of complex analysis. One of the outcomes of such analyses concerns the non-existence of linear recurrences with polynomial coefficients annihilating these sequences, and, accordingly, the non-existence of linear differential equations with polynomial coefficients annihilating their generating functions. In particular, the corresponding generating functions are transcendental. Asymptotic estimates of certain finite difference sequences come out as a byproduct of the Lindelöf approach.
\end{abstract}

\section{Introduction}

There has been recently a surge of interest in methods for proving that certain sequences coming from analysis or combinatorics are non-holonomic. Recall that a sequence $\left(f_{n}\right)$

${ }^{*}$ This work was partially supported by CDG, BA-CA, AFFA, and the joint INRIA-Microsoft Research Laboratory. 
is holonomic, or P-recursive, if it satisfies a linear recurrence with coefficients that are polynomial (equivalently, rational) in the index $n$; that is,

$$
\sum_{k=0}^{d} p_{k}(n) f_{n-k}=0, \quad p_{k}(n) \in \mathbb{C}[n], \quad p_{0} \not \equiv 0 .
$$

Put otherwise, its generating function $f(z)$, called holonomic or $D$-finite, satisfies a linear differential equation with coefficients that are polynomial (equivalently, rational) in the variable $z$; that is, with $f(z):=\sum_{n \geqslant 0} f_{n} z^{n}$,

$$
\sum_{k=0}^{e} q_{k}(z) \frac{\mathrm{d}^{k}}{\mathrm{~d} z^{k}} f(z)=0, \quad q_{k}(z) \in \mathbb{C}[z], \quad q_{e} \not \equiv 0 .
$$

Within combinatorics, the holonomic framework has been largely developed by Stanley, Zeilberger, Lipshitz, and Gessel [18, 25, 26, 35, 44], who provided a rich set of closure properties satisfied by the holonomic class. Since the class of holonomic functions contains all algebraic functions, establishing that a sequence is non-holonomic can in particular be regarded as a strong transcendence result for its generating function.

In recent years, proofs have appeared of the non-holonomic character of sequences such as

$$
\begin{array}{rllll}
\log n, & \sqrt{n}, & n^{n}, \quad \frac{1}{H_{n}}, & \varpi_{n}, \quad \log \log n, \quad \zeta(n), \\
\sqrt{n !}, & \arctan (n), \quad \sqrt{n^{2}+1}, \quad \mathrm{e}^{\mathrm{e}^{1 / n}}
\end{array}
$$

where $H_{n}$ is a harmonic number, $\varpi_{n}$ represents the $n$th prime number, and $\zeta(s)$ is the Riemann zeta function. The known proofs range from elementary [16] to algebraic and analytic $[2,10,22,23]$. The present paper belongs to the category of complex-analytic approaches and it is, to a large extent, a sequel to the paper [10].

Keeping in mind that a univariate holonomic function can have only finitely many singularities, we enunciate the following general principle.

Holonomicity criterion. The shape of the asymptotic expansion of a holonomic function at a singularity $z_{0}$ is strongly constrained, as it can only involve, in sectors of $\mathbb{C}$, (finite) linear combinations of "elements" of the form

$$
\exp \left(P\left(Z^{-1 / r}\right)\right) Z^{\alpha} \sum_{j=0}^{\infty} Q_{j}(\log Z) Z^{j s}, \quad Z:=\left(z-z_{0}\right),
$$

with $P$ a polynomial, $r$ an integer, $\alpha$ a complex number, $s$ a rational of $\mathbb{Q}_{>0}$ and the $Q_{j}$ a family of polynomials of uniformly bounded degree. (For an expansion at infinity, change $Z$ to $Z:=1 / z$.) Therefore, any function whose asymptotic structure at a singularity (possibly infinity) is incompatible with elements of the form (1) must be non-holonomic. 
Equation (1) is a paraphrase of the classical structure theorem for solutions of linear differential equations with meromorphic coefficients [20, 39]; see also Theorem 2 of [10] and the surrounding comments.

What the three of us did in [10] amounts to implementing the principle above, in combination with a basic Abelian theorem. Functional expansions departing from (1) can then be generated, by means of such a theorem, from corresponding terms in the asymptotic expansions of sequences: for instance, quantities such as

$$
\log \log n, \quad \frac{1}{\log n}, \quad \sqrt{\log n}, \quad \mathrm{e}^{\sqrt{\log n}}, \quad \ldots
$$

constitute forbidden "elements" in expansions of holonomic sequences - hence their presence immediately betrays a non-holonomic sequence. In proving the non-holonomic character of the sequences $(\log n)$ and $(\sqrt{n})$, we could then simply observe in [10] that the $n$th order differences (this is a holonomicity-preserving transformation) involve $\log \log n$ and $1 / \log n$ in an essential way.

What we do now, is to push the method further, but in another direction, namely, that of Lindelöf representations of generating functions. Namely, for a suitable "coefficient function" $\phi(s)$, one has

$$
\sum_{n=1}^{\infty} \phi(n)(-z)^{n}=-\frac{1}{2 \mathrm{i} \pi} \int_{1 / 2-\mathrm{i} \infty}^{1 / 2+\mathrm{i} \infty} \phi(s) z^{s} \frac{\pi}{\sin \pi s} \mathrm{~d} s,
$$

where the left side is, up to an alternating sign, the generating function of the sequence $(\phi(n))$. Based on representations of type (2), we determine directly the asymptotic behaviour at infinity of the generating functions of several sequences given in closed form, and detect cases that contradict (1), hence entail the non-holonomicity of the sequence $(\phi(n))$.

Here are typical results that can be obtained by the methods we develop.

Theorem 1. (i) The sequences $\mathrm{e}^{c n^{\theta}}$, with $c, \theta \in \mathbb{R}$, are non-holonomic, except in the trivial cases $c=0$ or $\theta \in\{0,1\}$. In particular,

$$
\mathrm{e}^{\sqrt{n}}, \quad \mathrm{e}^{-\sqrt{n}}, \quad \mathrm{e}^{1 / n}, \quad \mathrm{e}^{-1 / n}
$$

are non-holonomic.

(ii) The sequences

$$
\frac{1}{2^{n} \pm 1}, \quad \frac{1}{n !+1}, \quad \Gamma(n \sqrt{2}), \quad \frac{\Gamma(n \sqrt{2})}{\Gamma(n \sqrt{3})}, \quad \Gamma(n \mathrm{i}), \quad \frac{1}{\zeta(n+2)}
$$

are also non-holonomic.

The non-holonomicity of the sequences in (3) also appears in the article by Bell et al. [2], where it is deduced from an elegant argument involving Carlson's Theorem combined with the observation that $\phi(s)=\mathrm{e}^{c s^{\theta}}$ is non-analytic and non-polar at 0 . The 
results of [2] do usually not, however, give access to the cases where the function $\phi(s)$ is either meromorphic throughout $\mathbb{C}$ or entire. In particular, they do not seem to yield the non-holonomic character of the sequences listed in (4), except for the first one. (Indeed, 1/(2 \pm 1$)$ has been dealt with in [2] by an extension of the basic method of that paper. Moreover, any potential holonomic recurrence for $1 /\left(2^{n} \pm 1\right)$ can be refuted by an elementary limit argument, communicated by Frédéric Chyzak and transcribed in [17, Proposition 1.2.2].)

A great advantage of the Lindelöf approach is that it leads to precise asymptotic expansions that are of independent interest. For instance, in $\S 2.2$ below we will encounter the expansion

$$
\sum_{n \geqslant 1} \frac{(-z)^{n}}{n !+1} \sim-\sum_{k \geqslant 1} \frac{\pi}{\sin \pi s_{k}} \frac{1}{\Gamma^{\prime}\left(s_{k}+1\right)} z^{s_{k}}, \quad z \rightarrow \infty
$$

where

$$
\left(s_{k}\right)_{k \geqslant 1} \approx(-3.457,-3.747,-5.039,-5.991, \ldots)
$$

are the solutions of $\Gamma(s+1)=-1$. This formula features a remarkable structural difference to the superficially resembling function $\sum_{n \geqslant 1}(-z)^{n} / n !=\exp (-z)-1$.

Plan of the paper. The general context of Lindelöf representations is introduced in Section 1; in particular, Theorem 2 of that section provides detailed conditions granting us the validity of (2). As we show next, these representations make it possible to analyse the behaviour of generating functions towards $+\infty$, knowing growth and singularity properties of the coefficient function $\phi(s)$ in the complex plane. The global picture is a general correspondence of the form:

location $s_{0}$ of singularity of $\phi(s) \longrightarrow \quad$ singular exponent of $F(z)$ nature of singularity of $\phi(s) \longrightarrow$ logarithmic singular elements in $F(z)$.

(This is, in a way, a dual situation to singularity analysis $[11,13]$.) Precisely, three major cases are studied here; see Figure 2 below for a telegraphic summary.

- Polar singularities. When $\phi(s)$ can be extended into a meromorphic function, the asymptotic expansion of the generating function of the sequence $(\phi(n))$ at infinity can be read off from the poles of $\phi(s)$. Section 2 gives a detailed account of the corresponding "dictionary", which is in line with early studies by Ford [15]. It implies the non-holonomicity of sequences such as $1 /\left(2^{n}-1\right), 1 /(n !+1), \Gamma(n \sqrt{2})$.

- Algebraic singularities. In this case, a singularity of exponent $-\lambda$ in the function $\phi(s)$ essentially induces a term of the form $(\log z)^{\lambda-1}$ in the generating function as $z \rightarrow$ $+\infty$. We show the phenomena at stake by performing a detailed asymptotic study of the generating functions of sequences such as $\mathrm{e}^{\sqrt{n}}$ in Section 3 , based on the use of Hankel contours. The non-holonomic character of the sequences $\mathrm{e}^{ \pm n^{\theta}}$ for $\left.\theta \in\right] 0,1[$ arises as a consequence. 


\begin{tabular}{ccc}
\hline \hline Coefficients $\phi(n)$ & $z \rightarrow \infty$ & $z \rightarrow-1$ \\
\hline $\mathrm{e}^{1 / n}$ & $-\frac{\mathrm{e}^{2 \sqrt{\log z}}}{2 \sqrt{\pi}(\log z)^{1 / 4}}$ & $\frac{1}{1+z}$ \\
\hline $\mathrm{e}^{-1 / n}$ & $-\frac{1}{\sqrt{\pi}}(\log z)^{-1 / 4} \cos \left(2 \sqrt{\log z}-\frac{1}{4} \pi\right)$ & $\frac{1}{1+z}$ \\
\hline $\mathrm{e}^{\sqrt{n}}$ & $-1-\frac{1}{\sqrt{\pi \log z}}$ & $\frac{\sqrt{\pi} \mathrm{e}^{-1 / 8}}{(1+z)^{3 / 2}} \exp \left(\frac{1}{4(1+z)}\right)$ \\
\hline $\mathrm{e}^{-\sqrt{n}}$ & $-1+\frac{1}{\sqrt{\pi \log z}}$ & $\mathrm{E}(1)+\mathrm{E}^{\prime}(1)(1+z)$ \\
\hline \hline
\end{tabular}

Figure 1: Asymptotic forms of $\mathrm{E}(z ; c, \theta)$, for representative parameter values.

- Essential singularities. The case of an essential singularity is illustrated by $\phi(s)=$ $\mathrm{e}^{ \pm 1 / s}$ : in Section 4 , we work out the asymptotic form of the generating function at infinity, based on the saddle-point method. In this way, we also obtain the nonholonomic character of sequences such as $\mathrm{e}^{ \pm 1 / n}$ by methods that constitute an alternative to those of [2].

As the discussion above suggests, the present article can also serve as a synthetic presentation of the use of Lindelof integrals in the asymptotic analysis of generating functions. The scope is wide as it concerns a large number of generating functions whose coefficients obey an "analytic law". This is a subject, which, to the best of our knowledge, has not been treated systematically in recent decades (Ford's monograph was published in 1936). In particular, the joint use of Lindelöf representations and of saddle points in Section 4, as well as the corresponding estimates relative to the family of functions

$$
\mathrm{E}(z ; c, \theta):=\sum_{n=1}^{\infty} \mathrm{e}^{c n^{\theta}}(-z)^{n}
$$

appear to be new. Figure 1 summarizes some special cases of the results we obtain for $\mathrm{E}(z ; c, \theta)$. Note that six essentially different expansions occur, depending on the parameter values and on the singularity we are interested in, and observe the surprising occurrence of subtle oscillations associated with $\mathrm{e}^{-1 / n}$.

In Section 5 we show that the technology we have developed also provides non-trivial estimates in the calculus of finite differences. Finally, Section 6 completes our investigation of the function $\mathrm{E}(z ; c, \theta)$, by working out its asymptotic behaviour near $z=-1$.

\section{Lindelöf Representations}

Lindelöf integrals provide a means to express a function, knowing an "explicit law" for its Taylor coefficients. Let $s \mapsto \phi(s)$ be a complex function that is analytic at all points 
of $\mathbb{R}_{>0}$; the (ordinary) generating function of the sequence of values $(\phi(n))$ will be taken here in its alternating form:

$$
F(z):=\sum_{n \geqslant 1} \phi(n)(-z)^{n}
$$

The function $\phi(s)$, which is typically given by an explicit expression, represents the "law" of the coefficients of $F(z)$ : it extrapolates the integer-indexed sequence $(\phi(n))$ to a domain of the complex numbers that must contain the half-line $\mathbb{R}_{\geqslant 1}$. The key idea is to introduce the Lindelöf integral

$$
\Lambda(z ; \mathcal{C}):=\frac{1}{2 \mathrm{i} \pi} \int_{\mathcal{C}} \phi(s) z^{s} \frac{\pi}{\sin (\pi s)} \mathrm{d} s,
$$

where $\mathcal{C}$ is a contour enclosing the points $1,2,3, \ldots$ and lying within the domain of analyticity of $\phi(s)$. Formally, as well as analytically (see Theorem 2 below), when $\phi(s)$ is well-behaved near the positive real line, a basic residue evaluation shows that, with $\epsilon(\mathcal{C})= \pm 1$ representing the orientation of $\mathcal{C}$,

$$
\Lambda(z ; \mathcal{C})=\epsilon(\mathcal{C}) \sum_{n \geqslant 1} \phi(n)(-z)^{n}
$$

since the residue of $\pi / \sin \pi s$ at $s=n$ equals $(-1)^{n}$. Thus, the Lindelöf integral (7) provides a representation of the generating function $F(z)$ of $(6)$. Then, suitable growth conditions on $\phi(s)$ enable us to preserve the validity of (8) under suitable deformations of the contour $\mathcal{C}$. We state:

Theorem 2 (Lindelöf integral representation). Let $\phi(s)$ be a function analytic in $\Re(s)>$ 0, satisfying the growth condition

(Growth) $|\phi(s)|<C \cdot \mathrm{e}^{A|s|}$ as $|s| \rightarrow \infty$ for some $\left.A \in\right] 0, \pi[$ and $C>0$,

in $\Re(s) \geqslant 1 / 2$. Then the generating function $F(z)=\sum_{n \geqslant 1} \phi(n)(-z)^{n}$ is analytically continuable to the sector $-(\pi-A)<\arg (z)<(\pi-A)$, where it admits the Lindelöf representation:

$$
\sum_{n \geqslant 1} \phi(n)(-z)^{n}=-\frac{1}{2 i \pi} \int_{1 / 2-\mathrm{i} \infty}^{1 / 2+\mathrm{i} \infty} \phi(s) z^{s} \frac{\pi}{\sin \pi s} \mathrm{~d} s .
$$

Proof. By the growth condition, we have $|\phi(n)|=\mathrm{O}\left(\mathrm{e}^{A n}\right)$, so that $F(z)$ is a priori analytic in the open disc $|z|<\mathrm{e}^{-A}$. The proof proceeds in three moves.

(i) Fix $z$ to be positive real and satisfying $z<\mathrm{e}^{-A}$. Define the (positively oriented) rectangle $\mathcal{R}[m, N]$, with $m, N \in \mathbb{Z}_{>0}$ by its opposite corners at $1 / 2-N$ i and $m+1 / 2+N$ i. With the notation (7), Cauchy's residue theorem provides

$$
\Lambda(z ; \mathcal{R}[m, N])=\sum_{n=1}^{m} \phi(n)(-z)^{n} .
$$


We first dispose of the two horizontal sides of this rectangle. For $s$ in the complex plane punctured by small discs of fixed radius centred at the integers, one has

$$
\left|\frac{\pi}{\sin \pi s}\right|=\mathrm{O}\left(\mathrm{e}^{-\pi|\Im(s)|}\right) \text {. }
$$

A consequence of this estimate is that the integrand in the Lindelöf representation decays exponentially with $N$ : for fixed $z \in] 0, \mathrm{e}^{-A}[$, we have

$$
\phi(s) z^{s} \frac{\pi}{\sin \pi s}=\mathrm{O}\left(\mathrm{e}^{A N} \mathrm{e}^{-\pi N}\right),
$$

so that the integral along the two horizontal sides of $\mathcal{R}[m, N]$ is vanishingly small. One can accordingly let $N$ tend to $+\infty$, which gives in the limit

$$
\Lambda(z ; \mathcal{R}[m, \infty])=\sum_{n=1}^{m} \phi(n)(-z)^{n},
$$

meaning that a partial sum of $F(z)$ is expressed as the difference of two integrals along vertical lines.

(ii) We next let $m$ tend to infinity. With $z$ still a fixed positive quantity satisfying $z=\mathrm{e}^{-B}$ for some $B>A$ and $s=m+1 / 2+\mathrm{i} t$, we have, for some constants $K, K^{\prime}$

$$
\begin{aligned}
\left|\phi(s) z^{s} \frac{\pi}{\sin \pi s}\right| & <K \exp \left[A \sqrt{(m+1 / 2)^{2}+t^{2}}\right] \mathrm{e}^{-B m} \mathrm{e}^{-\pi|t|} \\
& <K^{\prime} \mathrm{e}^{(A-B) m} \mathrm{e}^{(A-\pi)|t|} .
\end{aligned}
$$

Thus, as a function of $m$, the Lindelöf integral is $\mathrm{O}\left(\mathrm{e}^{(A-B) m}\right)$. This shows that the contribution to the integral (7) arising from the rightmost vertical side of $\mathcal{R}[m,+\infty]$ is vanishingly small. Letting $m$ tend to infinity then yields the representation (9) in the limit (upon taking into account the change of sign due to orientation).

(iii) Finally, the convergence of the integral persists, for any real $z>0$, given the growth condition on $\phi$ : this ensures that $F(z)$ is analytic at all points of the positive real line. Furthermore, for $z=r \mathrm{e}^{\mathrm{i} \vartheta}$ and $s=1 / 2+\mathrm{i} t$, we have

$$
\left|z^{s}\right|=\left|\left(r \mathrm{e}^{\mathrm{i} \vartheta}\right)^{1 / 2+\mathrm{i} t}\right|=r^{1 / 2} \mathrm{e}^{-t \vartheta}
$$

so that the Lindelöf integral (9) remains convergent in the stated sector, where it provides the analytic continuation of $F(z)$.

This theorem was familiar to analysts about a century ago: it forms the basis of Chapter V of Lindelöf's treatise [24] dedicated to "prolongement analytique des séries de Taylor" and published in 1905; it underlies several chapters of W. B. Ford's monograph [15] relative to "The asymptotic developments of functions defined by Maclaurin series", first published in 1936. Lindelöf representations are also central in several works of Wright [41, 43] about generalizations of the exponential and Bessel functions. Last but not least, this circle of ideas can also provide a basis to Ramanujan's "Master Theorem" [3, pp. 298-323], as brilliantly revealed by Hardy in [19, Ch. XI]. (We propose to return to properties of the associated "magic duality" in another study.) 


\section{Sequences with Polar Singularities}

The original purpose of Lindelöf representations was to provide for analytic continuation properties. For instance, as a consequence of Theorem 2, generalized polylogarithms, such as

$$
\operatorname{Li}_{1 / 2,0}(z)=\sum_{n \geqslant 1} \frac{z^{n}}{\sqrt{n}}, \quad \operatorname{Li}_{0,1}(z)=\sum_{n \geqslant 1} \log n z^{n},
$$

are continuable into functions analytic in the complex plane slit along the ray from 1 to $+\infty$; see for instance $[9,15]$. Another fruitful corollary of the representations is the possibility of obtaining asymptotic expansions. In this section, we examine the simple case of generating functions whose coefficients admit a meromorphic lifting to $\mathbb{C}$.

\subsection{Polar singularities}

The following lemma ${ }^{1}$ is used throughout Ford's monograph [15, Ch. 1].

Lemma 1 (Ford's Lemma, polar case). Assume that $\phi(s)$ satisfies the conditions of Theorem 2. Assume that it is meromorphic in $\Re(s) \geqslant-B$ and analytic at all points of $\Re(s)=-B$. Assume finally that the growth condition (Growth) extends to the larger half-plane $\Re(s) \geqslant-B$. Then, the generating function $F(z)$ admits, as $z \rightarrow+\infty$, an asymptotic expansion of the form

$$
F(z)=-\sum_{1 / 2>\Re\left(s_{0}\right)>-B} \operatorname{Res}\left(\frac{\pi}{\sin \pi s} \phi(s) z^{s} ; s=s_{0}\right)+\mathrm{O}\left(z^{-B}\right), \quad z \rightarrow+\infty,
$$

where Res is the residue operator and the sum comprises all poles of $\phi(s) / \sin \pi s$ that lie in the strip $-B<\Re(s)<1 / 2$.

Proof. Start from (9), push the line of integration to the left, and take residues into account. This gives directly the expansion (12).

The following observations are to be made concerning (12).

(i) A pole of $\phi(s) / \sin \pi(s)$ at $s_{0}$ and of order $\mu \geqslant 1$ gives rise to a residue which is the product of a monomial in $z$ and a polynomial in $\log z$ :

$$
z^{s_{0}} P(\log z), \quad \text { where } \quad \operatorname{deg}(P)=\mu-1 .
$$

Such poles may arise either from $\phi(s)$ or from $1 / \sin \pi s$ at $s=0,-1,-2, \cdots$. In the case where $\phi(s)$ has no pole at $s=-n$, the induced residue is of the form $\phi(-n) z^{-n}$.

(ii) Additional poles of $\phi(s)$ in the right half-plane can be covered by an easy extension of the lemma, as long as they have bounded real parts and are not located at integers.

\footnotetext{
${ }^{1}$ This lemma is of course closely related to its specialization to hypergeometric functions, of which great use had been made in early works of Barnes and Mellin; see [34] and [40, Ch. XIV].
} 


\begin{tabular}{lcc}
\hline \hline Point $s_{0}$ & $\phi(s)$ & $F(z)$ \\
\hline Regular point $[\S 1]$ & & \\
$s_{0}=-n, \phi(s)$ analytic & $\phi(-n)+\cdots$ & $(-1)^{n+1} \phi(-n) z^{-n}$ \\
\hline Polar singularity $[\S 2]$ & $\frac{1}{s-s_{0}}$ & $-\frac{\pi}{\sin \pi s_{0}} z^{s_{0}}$ \\
$s_{0} \notin \mathbb{Z}$, simple pole of $\phi(s)$ & $z^{s_{0}} P_{\mu-1}(\log z)$ \\
$s_{0}$ pole of order $\mu$ of $\phi(s) / \sin \pi s$ & &
\end{tabular}

Algebraic singularity [§3]

\begin{tabular}{lcc}
$s_{0} \notin \mathbb{Z}$ & $\frac{1}{\left(s-s_{0}\right)^{\lambda}}$ & $-\frac{\pi}{\sin \pi s_{0}} \frac{z^{s_{0}}(\log z)^{\lambda-1}}{\Gamma(\lambda)}$ \\
\hline Essential singularity [§4] & $\mathrm{e}^{+\left(s-s_{0}\right)^{\theta}}$ & $-K_{1} z^{s_{0}}(\log z)^{\frac{\theta}{2(1-\theta)}} \exp \left(K_{2}(\log z)^{\frac{\theta}{\theta-1}}\right)$ \\
$s_{0} \in \mathbb{C}, \theta<0$ & $\mathrm{e}^{-\left(s-s_{0}\right)^{\theta}}$ & $L_{1} z^{s_{0}}(\log z)^{\frac{\theta}{2(1-\theta)}} \exp \left(\left(L_{2}+\mathrm{i} L_{3}\right)(\log z)^{\frac{\theta}{\theta-1}}\right)$ \\
$s_{0} \in \mathbb{C}, \theta<0$ & &
\end{tabular}

Figure 2: Sample cases of the correspondence between local (regular or singular) elements of a function at a point $s_{0}$ and the main asymptotic term in the expansion of the generating function $F(z)$ at infinity.

(iii) As a consequence of Item $(i)$, poles farthest on the right contribute the dominant terms in the asymptotic expansion of $F(z)$ at $+\infty$.

(iv) The asymptotic expansions of type (12) hold in a sector containing the positive real line. (To see this, note that the Lindelöf representation remains valid for $z$ in such a sector and that the growth condition in an extended half-plane guarantees the validity of the residue computation leading to (12).)

\subsection{Non-Holonomicity Resulting from Polar Singularities}

We list now a few sequences that may be proved non-holonomic by means of Ford's lemma (Lemma 1) in conjunction with the non-holonomicity criterion based on (1). We restrict ourselves to prototypes; a large number of variations are clearly possible.

Proposition 1. The following sequences are non-holonomic (with $\mathrm{i}=\sqrt{-1}$ ):

$$
\left\{\begin{array}{rlrl}
f_{1, n} & =\frac{1}{1+n !}, & f_{2, n}=\Gamma(n \sqrt{2}), & f_{3, n}=\frac{\Gamma(n \sqrt{2})}{\Gamma(n \sqrt{3})} \\
f_{4, n}=\frac{1}{2^{n}-1}, & f_{5, n}=\Gamma(n \mathrm{i}), & f_{6, n}=\frac{1}{\zeta(n+2)}
\end{array}\right.
$$

Proof. ( $i$ ) First the sequences $f_{1, n}, f_{2, n}, f_{3, n}$ are treated as direct consequences of Lemma 1. 
For $f_{1, n}=1 /(1+n !)$, we observe that the extrapolating function $\phi(s)=1 /(1+\Gamma(s))$ is meromorphic in the whole of $\mathbb{C}$. Thus the basic argument of $[2$, Th. 7$]$ is not applicable. However, examination of the roots of the equation $\Gamma(s)=-1$ reveals that there are roots near

$-2.457024, \quad-2.747682,-4.039361,-4.991544,-6.001385, \quad-6.999801, \quad-8.000024, \quad-8.999997$,

and so on, in a way that precludes the possibility of these roots to be accommodated into a finite number of arithmetic progressions. (To see this, note that if $\Gamma(s)=-1$, then $\Gamma(1-s)=-\pi / \sin \pi s$ by Euler's reflection formula. As $s$ moves farther to the left, the quantity $\Gamma(1-s)$ becomes very large; thus $\sin \pi s$ must be extremely close to zero. Hence $s$ itself must differ from an integer $-k$ by a very small quantity, which is found to be $\sim(-1)^{k-1} / k$ ! , in accordance with the numerical data above.) When transposed to the asymptotic expansion of $F(z)$ at infinity by means of Ford's Lemma (Lemma 1), this can be recognized to contradict the holonomicity criterion (1). Indeed, the exponents of $Z=z-z_{0}$ that can occur in the singular expansion of a holonomic function are invariably to be found amongst a finite union of arithmetic progressions, each of whose common difference must be a rational number.

A similar reasoning applies to $f_{2, n}=\Gamma(n \sqrt{2}), f_{3, n}=\Gamma(n \sqrt{2}) / \Gamma(n \sqrt{3})$, and more generally $^{2} \Gamma(\alpha n)$, where $\alpha \in \mathbb{R} \backslash \mathbb{Q}$. For instance, in the case of $f_{2, n}$, we should consider $\phi(s)=\Gamma(s \sqrt{2}) / \Gamma(s)^{2}$, where the normalization by $\Gamma(s)^{2}$ ensures both the analyticity at 0 of $F(z)$ and the required growth conditions at infinity of Lemma 1. The poles of $\phi(s)$ are now nicely aligned horizontally in a single arithmetic progression, but their common difference $(1 / \sqrt{2})$ is an irrational number.

(ii) Next, for the sequences $f_{4, n}, f_{5, n}, f_{6, n}$, we can recycle the proof technique of Lemma 1, so as to allow for an infinity of poles in a fixed-width vertical strip (details omitted).

In the case of $f_{4, n}$, we are dealing with the function $\phi(s)=1 /\left(2^{s}-1\right)$, which is meromorphic in $\mathbb{C}$, and has infinitely many regularly spaced poles at $s=2 \mathrm{i} k \pi / \log 2$, with $k \in \mathbb{Z}$. The "dictionary" suggested by Figure 2 and Lemma 1 applies to the effect that $F(z)$ has an expansion involving infinitely many elements of the form $z^{2 \mathrm{i} k \pi / \log 2}$. A similar argument applies to $f_{5, n}=\Gamma($ in $)$ which now has a half line of regularly spaced, vertically aligned, poles on $\Re(s)=0$.

As another consequence (but not a surprise!), the sequence $f_{6, n}=1 / \zeta(n+2)$ is nonholonomic, since $\phi(s)=1 / \zeta(s+2)$ satisfies the growth conditions of Theorem 2 and the Riemann zeta function has infinitely many non-trivial zeros. Likewise, for $f_{4, n}$ and $f_{5, n}$, the expansion of $F(z)$ contains exponents with infinitely many distinct imaginary parts. (Non-holonomicity does not depend on the Riemann hypothesis.)

In summary, assuming meromorphicity of the coefficient function $\phi(s)$ in $\mathbb{C}$ accompanied by suitable growth conditions in half-planes, sequences of the form $(\phi(n))$ are bound

\footnotetext{
${ }^{2}$ The corresponding generating functions are related to classical Mittag-Leffler and Wright functions [41, 42, 43]. They arise for instance in fractional evolution equations [28] and in the stable laws of probability theory $[8, \S$ XVII.6].
} 
to be non-holonomic as soon as the set of of poles of $\phi(s)$ is not included in a finite union of arithmetic progressions with rational common differences. In a way this extends the results of $[4,5]$ to cases of functions that are not entire.

\section{Sequences with Algebraic Singularities}

\subsection{Analogue of Ford's Lemma}

Ford also discusses the case where $\phi(s)$ has algebraic singularities. In this situation, it is no longer possible to move the contour of integration past singularities; Hankel contour integrals replace the residues of (12), and only expansions in descending powers of $\log z$ (rather than z) are obtained [15, Ch. III]. See Figure 2 for an aperçu. We first define the kind of singularities that can be handled.

Definition 1. The function $\phi(s)$ is said to have a singularity of algebraic type $(\lambda, \theta, \psi)$ at $s_{0}$ if a local expansion of the form

$$
\phi(s)=\left(s-s_{0}\right)^{-\lambda} \psi\left(\left(s-s_{0}\right)^{\theta}\right)
$$

holds in a slit neighborhood of $s_{0}$, where $\lambda \in \mathbb{C}, \Re(\theta)>0$, and

$$
\psi(s)=\sum_{k \geqslant 0} p_{k} s^{k}
$$

is analytic at zero.

To state the full result, we denote by $b_{j}\left(s_{0}\right)$ the coefficients in the expansion

$$
\frac{\pi}{\sin \pi s}=\sum_{j \geqslant-1} b_{j}\left(s_{0}\right)\left(s-s_{0}\right)^{j}, \quad s_{0} \in \mathbb{C} .
$$

Note that $b_{-1}\left(s_{0}\right)=0$ for $s_{0} \notin \mathbb{Z}$, while for $n \in \mathbb{Z}$, we have

$$
b_{-1}(n)=(-1)^{n}, \quad b_{2 k}(n)=0, \quad b_{2 k-1}(n)=(-1)^{n}\left(2-2^{2-2 k}\right) \zeta(2 k) .
$$

Lemma 2 (Ford's Lemma, algebraic case). Suppose that $\phi(s)$ is analytic throughout $\mathbb{C}$, except for finitely many singularities of algebraic type $\left(\lambda_{i}, \theta_{i}, \psi_{i}\right)$ at $s_{i}, i=1, \ldots, M$. The $m$-th branch cut should be at an angle $\left.\omega_{m} \in\right]-\frac{1}{2} \pi, 0[\cup] 0, \frac{1}{2} \pi[$ with the negative real axis, and the cuts may not intersect each other or the set $\mathbb{Z}_{>0}$. We impose the growth condition (Growth) with

$$
A<\pi \min _{1 \leqslant m \leqslant M}\left|\sin \omega_{m}\right|
$$

Assume furthermore that the singularities $s_{m}$ are sorted so that $\Re\left(s_{1}\right)=\cdots=\Re\left(s_{N}\right)>$ $\Re\left(s_{N+1}\right) \geqslant \cdots$, for some $1 \leqslant N \leqslant M$. Then $F(z)$ has the asymptotic expansion

$$
\begin{aligned}
F(z) \sim \sum_{0 \leqslant n \leqslant n_{\max }}(-1)^{n+1} \phi(-n) z^{-n} & \\
& \quad-\sum_{m=1}^{N} z^{s_{m}} \sum_{\substack{k \geqslant 0 \\
j \geqslant-1}} \frac{p_{m, k} b_{j}\left(s_{m}\right)}{\Gamma\left(-\theta_{m} k-j+\lambda_{m}\right)}(\log z)^{-\theta_{m} k-j+\lambda_{m}-1},
\end{aligned}
$$

THE ELECTRONiC JOURNAL OF COMBINATORICS 17 (2010), \#R3 


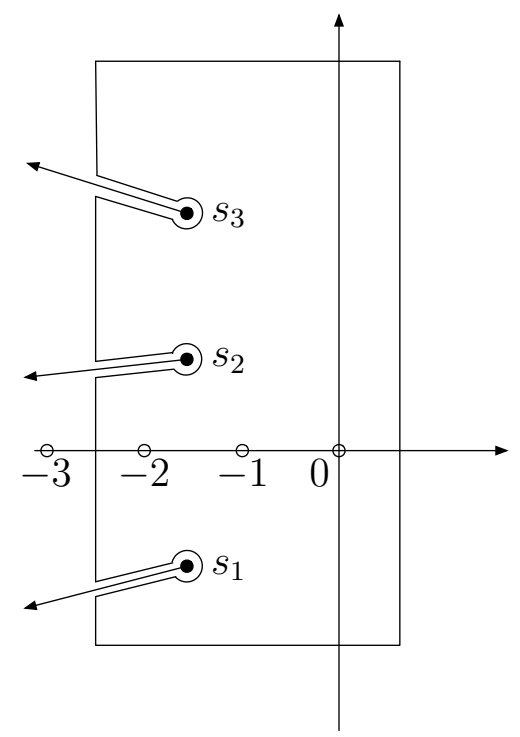

Figure 3: A rectangular integration contour embracing branch cut singularities.

where the $p_{m, k}$ are the coefficients in the expansion of $\psi_{m}$ at $s_{m}$, in the sense of (15), and the summation range of the first sum in (18) is determined by

$$
n_{\max }= \begin{cases}-\Re\left(s_{1}\right) & \Re\left(s_{1}\right) \in \mathbb{Z} \text { and } s_{m} \notin \mathbb{Z} \text { for } 1 \leqslant m \leqslant N \\ \left\lceil-\Re\left(s_{1}\right)\right\rceil-1 & \text { otherwise. }\end{cases}
$$

The variable $z$ may tend to infinity in any sector with vertex at zero that avoids the negative real axis.

Proof. The statement assembles and generalizes results by Barnes [1] and Ford [15], who treat the case $\theta_{m}=1$ (the latter reference offers a very detailed discussion). Extending the integration contour as usual (see Figure 3), we find the expansion

$$
F(z)+\frac{1}{2 \mathrm{i} \pi} \sum_{m=1}^{M} \int_{\mathcal{H}_{m}} \phi(s) z^{s} \frac{\pi}{\sin \pi s} \mathrm{~d} s \sim \sum_{\substack{n \geqslant 0 \\-n \notin\left\{s_{1}, \ldots, s_{M}\right\}}}(-1)^{n+1} \phi(-n) z^{-n},
$$

where $\mathcal{H}_{m}$ is a narrow Hankel-type contour, positively oriented and embracing the $m$-th branch cut. Suppose first that $M=1, s_{1}=0$, set $b_{j}:=b_{j}(0), p_{k}:=p_{1, k}$ (see (16) and (15)), and drop the index of $\psi_{1}, \lambda_{1}$, etc. In a slit neighborhood of zero, we then have

$$
\phi(s) \frac{\pi}{\sin \pi s}=s^{-\lambda} \sum_{\substack{k \geqslant 0 \\ j \geqslant-1}} p_{k} b_{j} s^{\theta k+j} .
$$

We first deal with a truncation

$$
T_{K}(s):=s^{-\lambda} \sum_{\substack{k \geqslant 0, j \geqslant-1 \\ \theta k+j<K}} p_{k} b_{j} s^{\theta k+j}
$$


of this expansion. Plugging $T_{K}$ in (20) and substituting $y=-s \log z$, we then evaluate the integral termwise by Hankel's formula for the Gamma function:

$$
-\frac{1}{2 \mathrm{i} \pi} \int_{-\mathcal{H}} \mathrm{e}^{-y}(-y)^{\eta} \mathrm{d} y=\frac{1}{\Gamma(-\eta)}, \quad \eta \in \mathbb{C} .
$$

We thus obtain

$$
\begin{aligned}
\frac{1}{2 \mathrm{i} \pi} \int_{\mathcal{H}} z^{s} T_{K}(s) \mathrm{d} s & =-\sum \frac{p_{k} b_{j}}{2 \mathrm{i} \pi}(\log z)^{-\theta k-j+\lambda-1} \int_{-(\log z) \mathcal{H}} \mathrm{e}^{-y}(-y)^{\theta k+j-\lambda} d y \\
& =\sum_{\substack{k \geqslant 0, j \geqslant-1 \\
\theta k+j<K}} \frac{p_{k} b_{j}}{\Gamma(-\theta k-j+\lambda)}(\log z)^{-\theta k-j+\lambda-1} .
\end{aligned}
$$

We now have to show that

$$
\int_{\mathcal{H}} z^{s}\left(\phi(s) \frac{\pi}{\sin \pi s}-T_{K}(s)\right) \mathrm{d} s=\mathrm{O}\left((\log z)^{-K+\lambda-1}\right) .
$$

For $s$ inside the circle of convergence of $\psi$, we can expand $\phi(s) \pi / \sin \pi s$, substitute $y=$ $-s \log z$, and estimate the series tail $\sum_{\theta k+j \geqslant K}$ by the triangle inequality.

To treat the remaining part of the contour $\mathcal{H}$, we pick a point $w$ on either of the rectilinear portions of $\mathcal{H}$, and lying inside the circle of convergence of $\psi$. By taking $\mathcal{H}$ narrow enough, both rays of $\mathcal{H}$ will admit such a point with $\Re(w)<0$. Then the portion $\int_{w}^{-\infty}$ of the integral in (21) is smaller than

$$
\mathrm{e}^{\Re(w \log z)} \int_{w}^{-\infty} \mathrm{e}^{\Re((s-w) \log z)}\left(C+|s|^{|\lambda|} \sum_{\theta k+j<K}\left|p_{k} b_{j}\right||s|^{\theta k+j}\right) \mathrm{d} s,
$$

where the boundedness of $|\phi(s) \pi / \sin \pi s|<C$ results from our growth assumption on $\phi(s)$ and the fact that $\sin \pi s=\mathrm{O}(\exp (-\pi|\Im(s)|))$. The integral in (22) converges, and

$$
\mathrm{e}^{\Re(w \log z)}=\mathrm{O}\left(|z|^{\Re(w)}\right)
$$

which is negligible in the logarithmic scale of the problem, as $\Re(w)<0$.

This completes the proof in the special case $M=1, s_{1}=0$. The full result follows from the special case by performing the substitutions $s \mapsto s+s_{m}$ in (20). Deleting redundant terms in the resulting expansion then yields (18). Note that in the first case of (19) we must include the contribution of the pole at $s=\Re\left(s_{1}\right)$ in (18), which gives rise to the summand $n=-\Re\left(s_{1}\right)$. Otherwise, we need to consider only the poles whose real part is larger than $\Re\left(s_{1}\right)$.

We make the following remarks concerning Lemma 2.

(i) Clearly, the statement extends to functions $\phi(s)$ having both poles and algebraic singularities. In fact the expansion (18) essentially remains valid then, as the reciprocal of the Gamma function vanishes at the non-positive integers. For instance, 
if $s_{m} \notin \mathbb{Z}$ is a simple pole, then only the summand $k=j=0$ of the inner sum remains, which is in line with Lemma 1 . The dominating singularities $s_{1}, \ldots, s_{N}$ that enter the expansion (18) must then not only comprise the rightmost algebraic singularities, but also the poles whose real parts are equal to theirs or greater.

(ii) We disallow horizontal branch cuts in the lemma, in order to take advantage of the exponential decrease of $\pi / \sin \pi s$ along vertical lines. If a horizontal cut is present, and $\phi(s)$ stays bounded near the cut, the result persists.

(iii) There is a slight error in the statement of [15, §5]: Ford assumes that his function $P$, which corresponds to our $\phi(-s) \pi s / \sin \pi s$, is bounded in a right half-plane. This is usually too restrictive, due to the poles of $1 / \sin \pi s$, and is in fact not satisfied by the application in $[15, \S 6]$.

(iv) By putting $\phi(s)=s^{-\lambda}$ in Lemma 2, we recover the classical expansion of the polylogarithm function at infinity $[15,32]$.

\subsection{Asymptotic Analysis of the Generalized Exponential E $(z ; c, \theta)$ when $\theta \in] 0,1[$.}

Recall that in this case $\phi(s)=\exp \left(c s^{\theta}\right)$, so that it is a straightforward application of Lemma 2 , with $M=1, \lambda=0$, and $\psi(s)=\mathrm{e}^{c s}$. In fact this example was our initial motivation to extend Ford's result to the case where $\theta_{m} \neq 1$. As for the branch cut of the function $s^{\theta}$, we may put it at any direction allowed by Lemma 2 . The resulting expansion is

$$
\mathrm{E}(z ; c, \theta) \sim-\sum_{\substack{k \geqslant 0 \\ j \geqslant-1}} \frac{c^{k} b_{j}(0)}{k ! \Gamma(-k \theta-j)}(\log z)^{-k \theta-j-1}, \quad z \rightarrow \infty,
$$

with $b_{j}(0)$ as given in (17). In particular, for the coefficient sequences $\mathrm{e}^{ \pm \sqrt{n}}$ we obtain

$$
\mathrm{E}\left(z ; c= \pm 1, \theta=\frac{1}{2}\right)=-1 \mp \frac{1}{\sqrt{\pi \log z}}+\mathrm{O}\left(\frac{1}{(\log z)^{3 / 2}}\right) .
$$

\subsection{Non-Holonomicity Resulting from Algebraic Singularities}

The estimates of Equation (23), when compared with the holonomicity criterion (1), immediately yield the non-holonomic character of simple sequences involving the exponential function, such as $\mathrm{e}^{ \pm \sqrt{n}}$ in Theorem 1 . More generally, we can state the following result.

Proposition 2. Suppose that $\phi(s)$ satisfies the assumptions of Lemma 2, and has a nonpolar singularity at $s_{1}$ with an expansion of the type (14). Then the sequence $(\phi(n))_{n \geqslant 1}$ is not holonomic.

Proof. This follows readily from the expansion (18) and the holonomicity criterion (1). Without loss of generality, we assume that $s_{1}$ has maximal real part among the non-polar 
singularities of $\phi(s)$. Now choose $k_{0} \geqslant 0$ such that $p_{k_{0}} \neq 0$ and $k_{0} \theta_{1}-\lambda_{1} \notin \mathbb{Z}$. (If there was no such $k_{0}$, then $s_{1}$ would either be a pole or no singularity at all.) It then suffices to pick some $j_{0} \geqslant-1$ with $b_{j_{0}} \neq 0$ to exhibit a logarithmic term with "forbidden", non-integral exponent and non-zero coefficient in (18).

Note that this proposition could also have been proved by the method of [2], based on Carlson's Theorem, but without the "constructive" feature of obtaining an asymptotic expansion of the associated generating functions.

\section{Sequences with Essential Singularities}

Going beyond the topics covered by Ford's treatise [15], we now investigate cases where the coefficient function $\phi(s)$ in $(2)$ has essential singularities. We do not aim at a general statement here, but instead restrict attention to the generalized exponential $\mathrm{E}(z ; c, \theta)$ from (5), with $\theta<0$. (In fact a general result encompassing both Theorems 3 and 4 below would probably be rather unwieldy.) This illustrates the use of Lindelöf representations in conjunction with the saddle-point method [7, 13]. The resulting asymptotic formulas (Theorems 3 and 4 below) will be immediately recognized to be incompatible with the structure formula of (1): in this way, the present section completes our proof of Theorem 1. (Two rather easy supplementary arguments, which serve to cover the whole range of parameter values, but involve only crude asymptotic analysis, are collected in Subsection 4.3.)

\subsection{Asymptotic Analysis of the Generalized Exponential E $(z ; c, \theta)$ when $c>0$ and $\theta<0$.}

In this section we determine the asymptotic behaviour of $\mathrm{E}(z)$ near infinity for positive $c$ and negative $\theta$. We present the analysis in the special case $c=1, \theta=-1$. The generalization to arbitrary $c>0$ and $\theta<0$ is then easy. We start once more from the Lindelöf integral representation

$$
\mathrm{E}(z ; 1,-1)=-\frac{1}{2 \mathrm{i} \pi} \int_{1 / 2-\mathrm{i} \infty}^{1 / 2+\mathrm{i} \infty} \mathrm{e}^{1 / s} z^{s} \frac{\pi}{\sin \pi s} \mathrm{~d} s .
$$

Neglecting the effect of $\pi / \sin \pi s$, the derivative

$$
\frac{\partial}{\partial s} \mathrm{e}^{1 / s} z^{s}=\left(\log z-s^{-2}\right) \mathrm{e}^{1 / s} z^{s}
$$

reveals a saddle point near $s=L^{-1 / 2}$, where $L:=\log |z|$. We accordingly move the integration contour in (24) to the left, obtaining

$$
\mathrm{E}(z ; 1,-1)=-\frac{1}{2 \mathrm{i} \pi} \int_{L^{-1 / 2}-\mathrm{i} \infty}^{L^{-1 / 2}+\mathrm{i} \infty} \mathrm{e}^{1 / s} z^{s} \frac{\pi}{\sin \pi s} \mathrm{~d} s .
$$




\begin{tabular}{l}
\hline \hline$s=a L^{-1 / 2}+b t, \quad a, b \in \mathbb{C} \backslash\{0\}, \quad|t|<L^{-\alpha}, \quad \frac{2}{3}<\alpha<\frac{3}{4}$ \\
$\pm \frac{1}{s}= \pm \frac{1}{a} L^{1 / 2} \pm \frac{b^{2}}{a^{3}} L^{3 / 2} t^{2} \mp \frac{b}{a^{2}} L t+\mathrm{O}\left(L^{2-3 \alpha}\right)$ \\
$\exp \left( \pm \frac{1}{s}\right)=\exp \left( \pm \frac{1}{a} L^{1 / 2} \pm \frac{b^{2}}{a^{3}} L^{3 / 2} t^{2} \mp \frac{b}{a^{2}} L t\right)\left(1+\mathrm{O}\left(L^{2-3 \alpha}\right)\right)$ \\
$\frac{\pi}{\sin \pi s}=\frac{1}{a} L^{1 / 2}\left(1+\mathrm{O}\left(L^{1 / 2-\alpha}\right)\right)$ \\
\hline$z^{s}=\exp \left(a L^{1 / 2}+b L t\right)\left(1+\mathrm{O}\left(L^{-1 / 2}\right)\right)$ \\
\hline \hline
\end{tabular}

Figure 4: Four elementary asymptotic expansions. The variable $L$ tends to $+\infty$, and the first line specifies the range of $s$ and the fixed parameters $a, b$, and $\alpha$.

We set $s=L^{-1 / 2}+\mathrm{i} t$. The main contribution to the integral arises near $t=0$, say for $|t|<L^{-\alpha}$, where it will turn out that $\frac{2}{3}<\alpha<\frac{3}{4}$ is a good choice. The expansions which we require to approximate the integrand around the saddle point are collected in Figure 4. (We will recycle them in the next section.) From these we obtain, provided that $\alpha>\frac{2}{3}$, the approximation

$$
\left.\frac{\mathrm{e}^{1 / s} z^{s}}{\sin \pi s}\right|_{s=L^{-1 / 2}+\mathrm{i} t}=\frac{1}{\pi} L^{1 / 2} \exp \left(2 L^{1 / 2}-L^{3 / 2} t^{2}\right) \cdot\left(1+\mathrm{O}\left(L^{2-3 \alpha}\right)\right) .
$$

This puts us in a position to evaluate the central part of the integral (25):

$$
\begin{aligned}
-\frac{1}{2 \mathrm{i}} \int_{L^{-1 / 2}-\mathrm{i} L^{-\alpha}}^{L^{-1 / 2}+\mathrm{i} L^{-\alpha}} & \frac{\mathrm{e}^{1 / s} z^{s}}{\sin \pi s} \mathrm{~d} s=-\frac{L^{1 / 2} \mathrm{e}^{2 L^{1 / 2}}}{2 \pi} \int_{-L^{-\alpha}}^{L^{-\alpha}} \mathrm{e}^{-L^{3 / 2} t^{2}} \mathrm{~d} t \\
& =-\frac{L^{1 / 2} \mathrm{e}^{2 L^{1 / 2}}}{2 \pi} \int_{-\sqrt{2} L^{3 / 4-\alpha}}^{\sqrt{2} L^{3 / 4-\alpha}} \frac{1}{\sqrt{2}} L^{-3 / 4} \mathrm{e}^{-r^{2} / 2} \mathrm{~d} r \\
& \sim-\frac{\mathrm{e}^{2 L^{1 / 2}}}{2 \sqrt{2} \pi L^{1 / 4}} \int_{-\infty}^{\infty} \mathrm{e}^{-r^{2} / 2} \mathrm{~d} r=-\frac{\mathrm{e}^{2 L^{1 / 2}}}{2 \sqrt{\pi} L^{1 / 4}},
\end{aligned}
$$

with a relative error of $\mathrm{O}\left(L^{2-3 \alpha}\right)$. In order to let the integration bounds of the Gaussian integral tend to infinity, we have assumed $\alpha<\frac{3}{4}$ here. The tails of the Gaussian integral then decrease exponentially in $L$.

In order to show that this is indeed the dominant part of the integral (25), it remains to prove that the portion of the integral from $i L^{-\alpha}$ to i $\infty$ (and thus, by symmetry, also from $-\mathrm{i} \infty$ to $\left.-\mathrm{i} L^{-\alpha}\right)$ grows more slowly.

First we consider $t=\Im(s) \geqslant 1$. In this range we have $\mathrm{e}^{1 / s}=\mathrm{O}(1)$, and

$$
\left|z^{s}\right|=\exp \left(L^{1 / 2}-t \arg z\right) \quad \text { and } \quad 1 / \sin \pi s=\mathrm{O}\left(\mathrm{e}^{-\pi t}\right)
$$

lead to the bound $\exp \left(L^{1 / 2}\right) \cdot \int_{1}^{\infty} \exp (-(\pi+\arg z) t) \mathrm{d} t$. To make the integral convergent, we assume that $z$ tends to infinity in a sector that does not contain the negative real axis. 
Now consider $L^{-\alpha} \leqslant t<1$. The factor $z^{s}$ is $\mathrm{O}\left(\exp \left(L^{1 / 2}\right)\right)$ there, and the estimate

$$
\left|\mathrm{e}^{1 / s}\right| \leqslant \mathrm{e}^{1 /|s|}=\mathrm{O}\left(\exp \left(L^{1 / 2}-\frac{1}{2} L^{3 / 2-2 \alpha}\right)\right)
$$

follows from evaluating $\mathrm{e}^{1 /|s|}$, which is a decreasing function of $\Im(s)$, at $s=L^{-1 / 2}+\mathrm{i} L^{-\alpha}$. Since

$$
1 / \sin \pi s=\mathrm{O}(1 / s)=\mathrm{O}\left(L^{\alpha}\right)
$$

we have established the tail estimate

$$
\int_{L^{-1 / 2}+\mathrm{i} L^{-\alpha}}^{L^{-1 / 2}+\mathrm{i} \infty} \mathrm{e}^{1 / s} z^{s} \frac{\pi}{\sin \pi s} \mathrm{~d} s=\mathrm{O}\left(L^{\alpha} \cdot \exp \left(2 L^{1 / 2}-\frac{1}{2} L^{3 / 2-2 \alpha}\right)\right),
$$

which grows slower than the absolute error in (26). Hence the asymptotic behaviour of $\mathrm{E}(z ; 1,-1)$ near infinity is

$$
\mathrm{E}(z ; 1,-1)=-\frac{\mathrm{e}^{2 \sqrt{\log z}}}{2 \sqrt{\pi}(\log z)^{1 / 4}}\left(1+\mathrm{O}\left((\log z)^{-1 / 4+\varepsilon}\right)\right), \quad z \rightarrow \infty .
$$

The error term follows from taking $\alpha \in] \frac{2}{3}, \frac{3}{4}\left[\right.$ close to $\frac{3}{4}$.

All steps of the previous derivation are easily extended, when $1 / s$ is replaced by $c s^{\theta}$, which yields the following result.

Theorem 3. Let $c>0$ and $\theta<0$ be real numbers. Then

$$
\mathrm{E}(z ; c, \theta)=-K_{1}(\log z)^{\frac{\theta}{2(1-\theta)}} \exp \left(K_{2}(\log z)^{\frac{\theta}{\theta-1}}\right)\left(1+\mathrm{O}\left((\log z)^{-\mu}\right)\right)
$$

as $z \rightarrow \infty$ in an arbitrary sector with vertex at zero that does not contain the negative real axis. The positive constants $K_{1}$ and $K_{2}$ are defined by

$$
K_{1}:=(2 \pi(1-\theta))^{-1 / 2}(-c \theta)^{\frac{1}{2(\theta-1)}} \quad \text { and } \quad K_{2}:=\left(1-\frac{1}{c \theta}\right)(-c \theta)^{\frac{1}{1-\theta}}
$$

and the exponent $\mu$ of the relative error estimate is

$$
\mu:= \begin{cases}\frac{\theta}{2(\theta-1)}-\varepsilon & \theta \geqslant-2 \\ \frac{1}{1-\theta} & \theta<-2\end{cases}
$$

with $\varepsilon$ an arbitrary positive real.

\subsection{Asymptotic Analysis of the Generalized Exponential E $(z ; c, \theta)$ when $c<0$ and $\theta<0$.}

We present the detailed proof for the parameter values $c=\theta=-1$. Then, the integrand of the Lindelöf integral

$$
\mathrm{E}(z ;-1,-1)=-\frac{1}{2 \mathrm{i} \pi} \int_{1 / 2-\mathrm{i} \infty}^{1 / 2+\mathrm{i} \infty} \mathrm{e}^{-1 / s} z^{s} \frac{\pi}{\sin \pi s} \mathrm{~d} s
$$



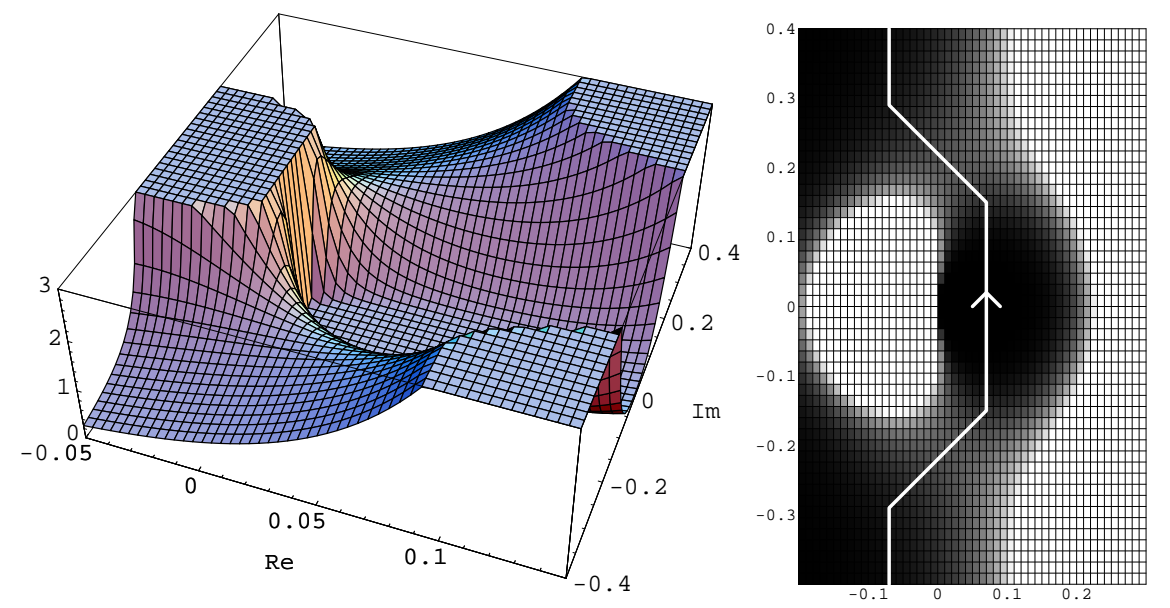

Figure 5: The landscape of $\left|z^{s} \mathrm{e}^{-1 / s} / \sin \pi s\right|$, where $z=10^{10}$, and the new integration contour crossing the two approximate saddle points.

has two saddle points, at $\pm \mathrm{i} L^{-1 / 2}$, roughly, which will induce an oscillating factor. The argument of the axis of the upper saddle point is [7]

$$
\frac{\pi}{2}-\left.\frac{1}{2} \arg \frac{\mathrm{d}^{2}}{\mathrm{~d} s^{2}}(-1 / s+L s)\right|_{s=\mathrm{i} L^{-1 / 2}}=\frac{3 \pi}{4}
$$

and that of the lower saddle point is $\frac{1}{4} \pi$. We choose an integration path that has two segments passing through these saddle points at an angle of $\pm \frac{1}{4} \pi$ with respect to the real axis and with length $\sqrt{2} L^{-\alpha}$, where $\alpha$ is yet to be chosen. The segments are joined by a vertical line, and extended by vertical lines towards \pm i $\infty$. Our path thus consists of the five segments (cf. Figure 5)

$$
\begin{array}{lll}
\mathcal{C}_{1}: & s=-L^{-\alpha}+\mathrm{i} t, & t \leqslant-L^{-1 / 2}-L^{-\alpha}, \\
\mathcal{C}_{2}: & s=-\mathrm{i} L^{-1 / 2}+(1+\mathrm{i}) t, & |t| \leqslant L^{-\alpha}, \\
\mathcal{C}_{3}: & s=L^{-\alpha}+\mathrm{i} t, & |t| \leqslant L^{-1 / 2}-L^{-\alpha}, \\
\mathcal{C}_{4}: & s=\mathrm{i} L^{-1 / 2}+(\mathrm{i}-1) t, & |t| \leqslant L^{-\alpha}, \\
\mathcal{C}_{5}: & s=-L^{-\alpha}+\mathrm{i} t, & t \geqslant L^{-1 / 2}+L^{-\alpha} .
\end{array}
$$

We will see that the exponent $\alpha$ must satisfy the same bounds as in the previous subsection, i.e. $\frac{2}{3}<\alpha<\frac{3}{4}$. For the segment $\mathcal{C}_{2}$, containing the lower saddle point, we again appeal to the expansions from Figure 4 and find

$$
\frac{z^{s} \mathrm{e}^{-1 / s}}{\sin \pi s}=\mathrm{i} \pi^{-1} L^{1 / 2} \exp \left(-2 \mathrm{i} L^{1 / 2}-2 L^{3 / 2} t^{2}\right) \cdot\left(1+\mathrm{O}\left(L^{2-3 \alpha}\right)\right) .
$$


Here we have set $s=-\mathrm{i} L^{-1 / 2}+(1+\mathrm{i}) t$, and assume that $\alpha>\frac{2}{3}$. Since

$$
\int_{-L^{-\alpha}}^{L^{-\alpha}} \exp \left(-2 L^{3 / 2} t^{2}\right) \mathrm{d} t \sim \frac{1}{\sqrt{2}} L^{-3 / 4} \int_{-\infty}^{\infty} \mathrm{e}^{-r^{2}} \mathrm{~d} r=\sqrt{\frac{\pi}{2}} L^{-3 / 4}
$$

for $\alpha<\frac{3}{4}$, we thus have

$$
\int_{\mathcal{C}_{2}} \frac{z^{s} \mathrm{e}^{-1 / s}}{\sin \pi s} \mathrm{~d} s=(\mathrm{i}-1)(2 \pi)^{-1 / 2} L^{-1 / 4} \mathrm{e}^{-2 \mathrm{i} L^{1 / 2}} \cdot\left(1+\mathrm{O}\left(L^{2-3 \alpha}\right)\right) .
$$

The contribution of the upper saddle point,

$$
\int_{\mathcal{C}_{4}} \frac{z^{s} \mathrm{e}^{-1 / s}}{\sin \pi s} \mathrm{~d} s=(\mathrm{i}+1)(2 \pi)^{-1 / 2} L^{-1 / 4} \mathrm{e}^{2 \mathrm{i} L^{1 / 2}} \cdot\left(1+\mathrm{O}\left(L^{2-3 \alpha}\right)\right),
$$

is similarly found. The dominant part of (28) is therefore

$$
\begin{aligned}
-\frac{1}{2 \mathrm{i}} \int_{\mathcal{C}_{2} \cup \mathcal{C}_{4}} \frac{z^{s} \mathrm{e}^{-1 / s}}{\sin \pi s} \mathrm{~d} s & =-\frac{1}{2 \mathrm{i}}\left(\int_{\mathcal{C}_{2}}-\overline{\int_{\mathcal{C}_{2}}}\right)=-\Im\left(\int_{\mathcal{C}_{2}} \frac{z^{s} \mathrm{e}^{-1 / s}}{\sin \pi s} \mathrm{~d} s\right) \\
& =-(2 \pi)^{-1 / 2} L^{-1 / 4}\left(\cos 2 L^{1 / 2}+\sin 2 L^{1 / 2}\right) \cdot\left(1+\mathrm{O}\left(L^{2-3 \alpha}\right)\right) \\
& =-\pi^{-1 / 2} L^{-1 / 4} \cos \left(2 L^{1 / 2}-\frac{1}{4} \pi\right) \cdot\left(1+\mathrm{O}\left(L^{2-3 \alpha}\right)\right) .
\end{aligned}
$$

It remains to bound the integrals over $\mathcal{C}_{1}, \mathcal{C}_{3}$, and $\mathcal{C}_{5}$. The portion of $\mathcal{C}_{5}$ with $t=$ $\Im(s) \geqslant 1$ is $\mathrm{O}\left(\exp \left(-L^{1-\alpha}\right)\right)$, by the same argument as in the case of one saddle point. Now consider the lower part of $\mathcal{C}_{5}$, where we have $L^{-1 / 2}+L^{-\alpha} \leqslant t<1$. The factor $\pi / \sin \pi s$ is of order $\mathrm{O}\left(L^{\alpha}\right)$. Also, it is easy to see that $|\exp (-1 / s)|$ is a decreasing function of $\Im(s)$ there. At the lower endpoint of $\mathcal{C}_{5}$, we estimate

$$
\exp (-1 / s)=\exp \left(L^{1-\alpha}-2 L^{3 / 2-2 \alpha}+\mathrm{o}(1)\right)
$$

Since we have $z^{s}=\mathrm{O}\left(\exp \left(-L^{1-\alpha}\right)\right)$ in $\mathcal{C}_{5}$, this segment contributes only $\exp \left(-2 L^{3 / 2-2 \alpha}+\right.$ $\mathrm{o}(1))$ to the integral. Finally, we examine the segment $\mathcal{C}_{3}$. The factor $|\exp (-1 / s)|$ is an increasing function of $|\Im(s)|$ there. Hence it suffices to estimate $\exp (-1 / s) z^{s}$ at the upper endpoint of $\mathcal{C}_{3}$, which is straightforward and shows that the integral over $\mathcal{C}_{3}$ is also negligible. This completes the tail estimate.

Equation (29) hence yields the result

$$
\mathrm{E}(z ;-1,-1)=-\frac{1}{\sqrt{\pi}}(\log z)^{-1 / 4} \cos \left(2 \sqrt{\log z}-\frac{1}{4} \pi\right)+\mathrm{O}\left((\log z)^{-1 / 2+\varepsilon}\right), \quad z \rightarrow \infty .
$$

The generalization to arbitrary negative parameters is as follows.

Theorem 4. Let $c<0$ and $\theta<0$ be real numbers. Then

$$
\begin{aligned}
& \mathrm{E}(z ; c, \theta)=A_{1} \exp \left(A_{2}(\log z)^{\frac{\theta}{\theta-1}}\right)(\log z)^{\frac{\theta}{2(1-\theta)}} \cos \left(A_{3}(\log z)^{\frac{\theta}{\theta-1}}+A_{4}\right) \\
&+\mathrm{O}\left(\exp \left(A_{2}(\log z)^{\frac{\theta}{\theta-1}}\right)(\log z)^{\frac{\theta}{2(1-\theta)}-\mu}\right)
\end{aligned}
$$


as $z \rightarrow \infty$ in an arbitrary sector with vertex at zero that does not contain the negative real axis. The constants are defined by

$$
\begin{array}{ll}
A_{1}=-(c \theta)^{\frac{1}{2(\theta-1)}} \sqrt{\frac{2}{\pi(1-\theta)}}, & A_{2}=\left(1-\theta^{-1}\right)(c \theta)^{\frac{1}{1-\theta}} \cos \frac{\pi}{1-\theta}, \\
A_{3}=\left(1-\theta^{-1}\right)(c \theta)^{\frac{1}{1-\theta}} \sin \frac{\pi}{1-\theta}, & A_{4}=\frac{\pi}{2(\theta-1)},
\end{array}
$$

so that $A_{2}$ is negative for $-1<\theta<0$, zero for $\theta=-1$, and positive for $\theta<-1$. The exponent $\mu$ is as in Theorem 3.

Proof. The general proof is very similar to the special case $c=\theta=-1$ (see above), upon taking into account the following comments. There might be more than two saddle points in general, but we have to consider only the ones that form a conjugate pair having the largest real part, which are (approximately) at $\exp ( \pm \mathrm{i} \pi /(1-\theta)) L^{1 /(\theta-1)}$. The saddle point axes have the arguments $\pm \frac{1}{2} \pi(2-\theta) /(1-\theta)$. When $-3 \leqslant \theta \leqslant-1$, the proof proceeds as above. The parameter $\alpha$, which governs the size of the two contour segments containing the saddle points, must satisfy

$$
\frac{3-\theta}{3(1-\theta)}<\alpha<\frac{2-\theta}{2(1-\theta)}
$$

For $\theta<-3$, there is one minor problem in the tail estimate: the decrease of

$$
\left|\exp \left(c s^{\theta}\right)\right|=\exp \left(c|s|^{\theta} \cos (\theta \arg (s))\right)
$$

does not hold on the whole of $\mathcal{C}_{5}$. In fact it is plausible that there are oscillations here if $|\theta|$ is large. What happens is that, moving upwards along $\mathcal{C}_{5}$, the quantity $\left|\exp \left(c s^{\theta}\right)\right|$ decreases up to a local minimum, at $s_{0}$ say. This results from elementary analysis; the argument of $s_{0}$ is $\arg \left(s_{0}\right)=2 \pi /(1-\theta)$. Now the integral over the portion of $\mathcal{C}_{5}$ below $s_{0}$ can be estimated as before, by taking into account the length of this part of the contour and the size of the integrand at the lower endpoint of $\mathcal{C}_{5}$. Above $s_{0}$, the crude estimate $\left|\exp \left(c s^{\theta}\right)\right| \leqslant \exp \left(-c|s|^{\theta}\right)$ suffices.

Finally, when $-1<\theta<0$, the contour has to be slightly adjusted, as the two saddle points at $\exp ( \pm \mathrm{i} \pi /(1-\theta)) L^{1 /(\theta-1)}$ have negative real part, and we may not push the contour over the singularity at zero. Instead of joining the two central segments $\mathcal{C}_{2}$ and $\mathcal{C}_{4}$ by a vertical line, we stretch each of them into the right half-plane, stopping at $\Re(s)=L^{-\alpha}$. Then we join them by a vertical line, whose upper endpoint we call $s_{1}$. The value $\left|\exp \left(c s^{\theta}\right)\right|$ decreases as we move on $\mathcal{C}_{4}$ to the right, until $\Re(s)=0$, from which point it increases. Moreover, as we move downwards from $s_{1}$, the quantity $\left|\exp \left(c s^{\theta}\right)\right|$ decreases until $\Im(s)=0$. Therefore, it suffices to check that the integrand at $s_{1}$ is of growth slower than the central part of the integral, a property that is easily checked to hold.

\subsection{Completion of the Proof of Theorem 1}

It remains to consider the parameter region $\theta>1$, which is not covered by our previous asymptotic estimates of $\mathrm{E}(z ; c, \theta)$. First, for parameter values $c<0,1<\theta$, which 
make $\mathrm{E}(z ; c, \theta)$ an entire function, a formula similar to (27) holds. The exponential growth order is the same as in (27), except for a different constant in place of $K_{2}$. In particular, the sequence $\mathrm{e}^{c n^{\theta}}$ is not holonomic for these parameter values either, as $\theta /(\theta-1) \neq 1$. This asymptotic property is a special case of a result due to Valiron [38], who used the Laplace method to investigate the behaviour of $\sum_{n \geqslant 0} \mathrm{e}^{-G(n)} x^{n}$ as $x \rightarrow \infty$, where $G$ is a smooth function that satisfies certain regularity conditions. (Valiron's conditions actually require $1<\theta \leqslant 2$, but his analysis is easily extended.)

Finally, in the remaining parameter range $c>0, \theta>1$, the sequence $\mathrm{e}^{c n^{\theta}}$ grows faster than any power of $n$ !, which is incompatible with the growth of any holonomic sequence. (In fact this observation shows that the (formal!) power series $\mathrm{E}(z ; c, \theta)$ does not even satisfy an algebraic differential equation [33].)

\section{$5 \quad$ Asymptotics of Finite Differences}

Beyond establishing non-holonomicity, the analysis near infinity of generating functions, such as $\mathrm{E}(z ; c, \theta)$, is also of interest in the estimation of finite differences and related combinatorial sums. Given a sequence $\left(f_{n}\right)$, we shall refer to the derived sequence

$$
D_{n}[f]:=\sum_{k=0}^{n}\left(\begin{array}{l}
n \\
k
\end{array}\right)(-1)^{k} f_{k}
$$

as the sequences of differences. (In the standard terminology, we have $D_{n}[f] \equiv(-1)^{n} \Delta^{n} f_{0}$; see $[21,29]$.) The relation between $f_{n}$ and $g_{n}:=D_{n}[f]$ is translated at generating function level by the relation

$$
g(z)=\frac{1}{1-z} f\left(-\frac{z}{1-z}\right)=\frac{1}{1-z}\left(f_{0}+F\left(\frac{z}{1-z}\right)\right),
$$

where $f(z), g(z)$ are the "standard" (i.e., non-alternating) generating functions

$$
f(z):=\sum_{n \geqslant 0} f_{n} z^{n}, \quad g(z):=\sum_{n \geqslant 0} g_{n} z^{n}
$$

and $F(z) \equiv f(-z)-f_{0}$ is the "alternating" generating function of (6).

Because of the alternation of signs in (31) and the fact that the binomial coefficients become almost as large as $2^{n}$, the asymptotic estimation of differences is usually a non-trivial task. Here, the "surprise" is the fact that, for many explicit and simple sequences $f_{n}$, the corresponding $g_{n}$ are much smaller than $2^{n}$ : huge cancellations occur in (31) (see, e.g., [12], for cases related to $f_{n}=n^{\alpha}, \log n$, and so on). For instance, with $f_{n}=\mathrm{e}^{1 / n}, n \geqslant 1$, we find

$$
g_{1} \doteq-2.71828, \quad g_{10} \doteq-8.03246, \quad g_{100} \doteq-20.4159, \quad g_{1000} \doteq-45.1379,
$$

and the sequence appears to grow rather slowly. For $f_{n}=\mathrm{e}^{\sqrt{n}}$, it even appears numerically to tend slowly to 0 . (For recent estimates relative to zeta values and inverse zeta values, 
see for instance, [14].) We now explain how a Lindelöf type of analysis can serve to quantify such phenomena.

The basic message of singularity analysis theory $[11,13,30]$ is that the behaviour of a sequence is (usually) detectable from the singularities of its generating function. Here, we should investigate the singularity of $g(z)$ at $z=1$. Now, this singularity is tightly coupled with the one of $(1-z) g(z)$, which by $(32)$ depends on the behaviour of $F(z)$ near $+\infty$. (This, by elementary properties of the conformal map $z \mapsto w=z /(1-z)$ and its inverse $w \mapsto z=w /(1+w)$.) Clearly, by considering specific analytic maps $\sigma(z)$ (here: $\sigma(z)=z /(1-z)$ ), a large number of seemingly hard alternating sums become asymptotically tractable.

Corollary 1. The differences of the sequences $\mathrm{e}^{ \pm \sqrt{n}}$ and $\mathrm{e}^{ \pm 1 / n}$ have the following asymptotic behaviour.

$$
\begin{aligned}
& \sum_{k=0}^{n}\left(\begin{array}{l}
n \\
k
\end{array}\right)(-1)^{k} \mathrm{e}^{ \pm \sqrt{k}} \sim-\frac{ \pm 1}{\sqrt{\pi \log n}}, \\
& \sum_{k=1}^{n}\left(\begin{array}{l}
n \\
k
\end{array}\right)(-1)^{k} \mathrm{e}^{1 / k} \sim-\frac{\mathrm{e}^{2 \sqrt{\log n}}}{2 \sqrt{\pi}(\log n)^{1 / 4}}, \\
& \sum_{k=1}^{n}\left(\begin{array}{l}
n \\
k
\end{array}\right)(-1)^{k} \mathrm{e}^{-1 / k}=-\frac{\cos \left(2 \sqrt{\log n}-\frac{1}{4} \pi\right)}{\sqrt{\pi}(\log n)^{1 / 4}}+\mathrm{o}\left((\log n)^{-1 / 4}\right) .
\end{aligned}
$$

Proof. Consider $f_{n}=\mathrm{e}^{ \pm \sqrt{n}}$, and define $g(z)$ as above. Then (23) implies

$$
g(z) \sim-\frac{ \pm 1}{\sqrt{\pi}} \frac{1}{1-z}\left(\log \frac{1}{1-z}\right)^{-1 / 2}, \quad z \rightarrow 1
$$

whence (33) follows by the appropriate transfer theorem $[11,13,30]$. In the cases $f_{n}=$ $\mathrm{e}^{ \pm 1 / n}$ (with $f_{0}=0$ ), the growth of the difference generating functions, say $g_{1}(z)$ and $g_{2}(z)$, can be determined by Theorems 3 and 4 . Note that both slowly varying and periodic functions can be subjected to singularity analysis $[11,13,37]$, so that the formulas

$$
\begin{aligned}
g_{2}(z) \sim & -\frac{1}{2 \sqrt{\pi}(1-z)} \exp \left(2\left(\log \frac{1}{1-z}\right)^{1 / 2}\right)\left(\log \frac{1}{1-z}\right)^{-1 / 4}, \\
g_{3}(z)= & -\frac{1}{\sqrt{\pi}(1-z)}\left(\log \frac{1}{1-z}\right)^{-1 / 4} \cos \left(2 \sqrt{\log \frac{1}{1-z}}-\frac{\pi}{4}\right) \\
& +\mathrm{o}\left(\frac{1}{1-z}\left(\log \frac{1}{1-z}\right)^{-1 / 4}\right),
\end{aligned}
$$

yield (34) and (35), respectively.

The estimate (34) bears a striking formal resemblance with the growth of the average value of the multiplicative partition function, which was found by Oppenheim [31] and 
Szekeres and Turán [36]. Indeed, only the sign and the exponent of $\log n\left(-\frac{3}{4}\right.$ instead of $\left.-\frac{1}{4}\right)$ differ.

As an application of Corollary 1, we note that Madsen [27] has considered generalized binomial distributions of the form

$$
\mathbb{P}[X=x]=\left(\begin{array}{l}
n \\
x
\end{array}\right) \sum_{j=0}^{n-x}\left(\begin{array}{c}
n-x \\
j
\end{array}\right)(-1)^{j} \pi_{x+j}, \quad x \in\{0, \ldots, n\},
$$

where, for instance, he sets $\pi_{k}=\exp \left((\log p) k^{a}\right)$ with $0 \leqslant a \leqslant 1$ and $0<p<1$. We can then describe, by an obvious extension of (33), the way the probability mass function behaves for large parameters $n$ :

$$
\mathbb{P}[X=x] \underset{n \rightarrow \infty}{\sim}\left\{\begin{array}{ll}
-\frac{\log p}{\Gamma(1-a)(\log n)^{a}} & x=0 \\
-\frac{a \log p}{x \Gamma(1-a)(\log n)^{a+1}} & x \geqslant 1
\end{array} .\right.
$$

In particular, there is no limit distribution, as $n \rightarrow \infty$.

\section{Behavior of $\mathrm{E}(z ; c, \theta)$ at its Dominating Singularity}

Although not related to non-holonomicity or the Ford-Lindelöf technique, it seems natural to complement the asymptotic results we have obtained for the function (5) by investigating its dominating singularity, located at $z=-1$. The asymptotic behaviour there is comparatively easy to determine. To begin with, for $\theta<0$ and any real $c$ we can rewrite

$$
\mathrm{E}(z ; c, \theta)=\sum_{n \geqslant 1} \sum_{k \geqslant 0} \frac{c^{k}}{k !} n^{k \theta}(-z)^{n}=\sum_{k \geqslant 0} \frac{c^{k}}{k !} \operatorname{Li}_{-k \theta}(-z), \quad|z|<1,
$$

as a sum of polylogarithms

$$
\operatorname{Li}_{\alpha}(z)=\sum_{n \geqslant 1} \frac{z^{n}}{n^{\alpha}}
$$

whose asymptotic behaviour at $z=1$ is known. The shape of the asymptotic expansion of $\mathrm{Li}_{\alpha}$ depends on whether $\alpha$ is an integer $[9,13]$.

Proposition 3. Let $c$ be a real number and $\theta$ be a negative real number. Then the asymptotic expansion of $\mathrm{E}(z ; c, \theta)$ at $z=-1$ is obtained by transporting the expansions of $\mathrm{Li}_{-k \theta}$ into (36).

Adding infinitely many asymptotic expansions termwise can be easily justified here by truncating the expansions and appealing to uniform convergence, which permits us to exchange limit and summation. For instance, if $\alpha \geqslant 1$ is an integer, we have

$$
\operatorname{Li}_{\alpha}(z)=\frac{(-1)^{\alpha}}{(\alpha-1) !} w^{\alpha-1}\left(\log w-H_{\alpha-1}\right)+\sum_{j \geqslant 0, j \neq \alpha-1} \frac{(-1)^{j}}{j !} \zeta(\alpha-j) w^{j}
$$


where $w=-\log z$ and $H_{\alpha-1}$ is a harmonic number. For the parameter values $c=1$, $\theta=-1$ we thus obtain

$$
\begin{aligned}
\mathrm{E}(z ; c=1, \theta=-1) & =\sum_{n \geqslant 1} \mathrm{e}^{1 / n}(-z)^{n}=\sum_{k \geqslant 0} \frac{1}{k !} \operatorname{Li}_{k}(-z) \\
& =\frac{1}{1+z}+\log \frac{1}{1+z}+C+\mathrm{O}\left((1+z) \log \frac{1}{1+z}\right),
\end{aligned}
$$

where $C=-1+\sum_{k \geqslant 2} \zeta(k) / k ! \approx 0.078189$.

We proceed to the case $c>0$ and $0<\theta<1$. For fixed $z$ inside the unit disk, the summands have a peak at some $n$ and then decrease rapidly, which makes the Laplace method a natural approach for estimating the sum. Our illustrative example is $c=1$ and $\theta=\frac{1}{2}$. The summands $\mathrm{e}^{\sqrt{n}}(-z)^{n}$ have their peak near $u:=\left\lfloor\frac{1}{4} v^{-2}\right\rfloor$, where $v:=$ $-\log |z|$. This leads to the lower bound $\exp \left(\frac{1}{4} v^{-1}\right)(1+\mathrm{O}(v))$, which was already noted by Borel [6, p. 69]. It is straightforward to determine the second order approximation of the summand near $n=u$, and to estimate the tails of the original sum and the second order approximation. What we find is

$$
\begin{aligned}
\mathrm{E}(z ; c=1, \theta & \left.=\frac{1}{2}\right)=\sum_{n \geqslant 1} \mathrm{e}^{\sqrt{n}}(-z)^{n} \\
& =\frac{\sqrt{\pi} \mathrm{e}^{-1 / 8}}{(1+z)^{3 / 2}} \exp \left(\frac{1}{4(1+z)}\right)\left(1+\mathrm{O}\left((1+z)^{1 / 2-\varepsilon}\right)\right), \quad z \rightarrow-1^{+} \text {in } \mathbb{R} .
\end{aligned}
$$

The (again straightforward) generalization from $\sqrt{n}$ to $c n^{\theta}$ reads as follows.

Proposition 4. Let $c$ be a positive real number, $0<\theta<1$, and $\varepsilon>0$. Then

$$
\mathrm{E}(z ; c, \theta)=C_{1}(1+z)^{\frac{2-\theta}{2(\theta-1)}} \exp \left(C_{2} v^{\frac{\theta}{\theta-1}}\right)\left(1+\mathrm{O}\left((1+z)^{\mu}\right)\right)
$$

as $z$ tends to $-1^{+}$in $\mathbb{R}$, where $v:=-\log |z|$. The constants $C_{1}, C_{2}, C_{3}$ are positive and given by

$$
\begin{aligned}
& C_{1}:=\sqrt{2 \pi}(1-\theta)^{-1 / 2}(c \theta)^{\frac{1}{2(1-\theta)}}, \quad C_{2}:=\frac{1-\theta}{\theta}(c \theta)^{\frac{1}{1-\theta}}, \\
& C_{3}:=(c \theta)^{\frac{1}{1-\theta}},
\end{aligned}
$$

and the exponent of the relative error estimate is

$$
\mu:=\min \left\{\frac{\theta}{2(1-\theta)}-\varepsilon, 1\right\} .
$$

Finally, we consider $c<0$ and $0<\theta<1$. Then the series

$$
\mathrm{E}(z ; c, \theta)=\sum_{n \geqslant 1} \mathrm{e}^{c n^{\theta}}(-z)^{n}
$$

converges at $z=-1$. Inside the unit circle we may differentiate it termwise arbitrarily many times, and the series thus obtained converge at $z=-1$, too. By Abel's convergence theorem, these values equal the limits of the derivatives as $z \rightarrow-1^{+}$. The (divergent) formal Taylor series of $\mathrm{E}(z)$ at $z=-1$ obtained in this way is an asymptotic series for the function [15, p. 30], which yields the following result. 
Proposition 5. Suppose that $c<0$ and $0<\theta<1$. Then

$$
\mathrm{E}(z ; c, \theta) \underset{z \rightarrow-1^{+}}{\sim} u_{0}+u_{1}(1+z)+u_{2}(1+z)^{2}+\ldots
$$

as $z$ tends to $-1^{+}$in $\mathbb{R}$, where the coefficients are given by

$$
u_{k}:=\frac{1}{k !} \lim _{z \rightarrow-1^{+}} \frac{\mathrm{d}^{k}}{\mathrm{~d} z^{k}} \mathrm{E}(z)=(-1)^{k} \sum_{n \geqslant 1}\left(\begin{array}{l}
n \\
k
\end{array}\right) \exp \left(c n^{\theta}\right) .
$$

Note that the series (38) does not converge in any neighborhood of $z=-1$, since Stirling's formula yields

$$
\left|u_{k}\right| \geqslant\left(\begin{array}{c}
n(k) \\
k
\end{array}\right) \mathrm{e}^{c n(k)^{\theta}} \gg k^{(1 / \theta-1-\varepsilon) k}
$$

where $n(k)=\left\lfloor(-k / c \theta)^{1 / \theta}\right\rfloor$ approximates the index of the largest summand in (39). Hence $z=-1$ is indeed a singularity.

\section{Conclusion}

We have revisited a classical method for the analytic continuation of power series beyond their disc of convergence, with the goal of obtaining asymptotic expansions and comparing them against the possible expansions of holonomic functions. Our estimates can be used as building blocks for the asymptotic analysis of more complicated functions than those we have explicitly mentioned. For instance, the expansion of functions such as

$$
\sum_{n \geqslant 0} \frac{\sqrt{n} \mathrm{e}^{\sqrt{n}}(-z)^{n}}{2^{n}+n^{2}}
$$

at $+\infty$ readily springs from Lemma 2 in $\S 3$, and series in the spirit of

$$
\sum_{n \geqslant 0} \mathrm{e}^{1 / n+1 / \sqrt{n}}(-z)^{n}
$$

can be analysed similarly to the ones in $\S 4.1$ and $\S 4.2$. As for proving non-holonomicity, our results compete with those of Bell et al. [2], who also deal with sequences having an analytic lifting. Roughly speaking, our approach is more versatile for meromorphic functions, equivalent in the algebraic case, and less flexible in the presence of essential singularities.

However, neither we nor Bell et al. [2] can show non-holonomicity of sequences whose extrapolating function is entire. For instance, we leave the non-holonomicity of sequences like $\cos (\sqrt{n})$ and $\cosh (\sqrt{n})$ as an open problem, since their analytic liftings, namely, $\cos (\sqrt{s})$ and $\cosh (\sqrt{s})$, have no singularity at a finite distance. 


\section{References}

[1] Barnes, E. W. The asymptotic expansion of integral functions defined by Taylor's series. Philosophical Transactions of the Royal Society of London. Series A 206 (1906), 249-297.

[2] Bell, J. P., Gerhold, S., Klazar, M., and Luca, F. Non-holonomicity of sequences defined via elementary functions. Annals of Combinatorics 12, 1 (2008), $1-16$.

[3] Berndt, B. C. Ramanujan's Notebooks, Part I. Springer Verlag, 1985.

[4] BÉzivin, J.-P., And Gramain, F. Solutions entières d'un système d'équations aux différences. Annales de l'institut Fourier 43, 3 (1993), 791-814.

[5] BÉzivin, J.-P., And Gramain, F. Solutions entières d'un système d'équations aux différences II. Annales de l'institut Fourier 43, 3 (1993), 791-814.

[6] Borel, É. Leçons sur les séries à termes positifs. In Collection de monographies sur la théorie des fonctions, publiée sous la direction de M. Émile Borel. GauthiersVillars, Paris, 1902.

[7] De Bruijn, N. G. Asymptotic Methods in Analysis. Dover, 1981. A reprint of the third North Holland edition, 1970 (first edition, 1958).

[8] Feller, W. An Introduction to Probability Theory and Its Applications, vol. 2. John Wiley, 1971.

[9] Flajolet, P. Singularity analysis and asymptotics of Bernoulli sums. Theoretical Computer Science 215, 1-2 (1999), 371-381.

[10] Flajolet, P., Gerhold, S., And Salvy, B. On the non-holonomic character of logarithms, powers, and the $n$th prime function. Electronic Journal of Combinatorics 11(2), A1 (2005), 1-16.

[11] Flajolet, P., and Odlyzko, A. M. Singularity analysis of generating functions. SIAM Journal on Algebraic and Discrete Methods 3, 2 (1990), 216-240.

[12] Flajolet, P., And Sedgewick, R. Mellin transforms and asymptotics: finite differences and Rice's integrals. Theoretical Computer Science 144, 1-2 (June 1995), $101-124$.

[13] Flajolet, P., And Sedgewick, R. Analytic Combinatorics. Cambridge University Press, 2009. 824 pages (ISBN-13: 9780521898065); also available electronically from the authors' home pages.

[14] Flajolet, P., And Vepstas, L. On differences of zeta values. Journal of Computational and Applied Mathematics 220, 1-2 (2008), 58-73.

[15] ForD, W. B. Studies on divergent series and summability and the asymptotic developments of functions defined by Maclaurin series, 3rd ed. Chelsea Publishing Company, 1960. (From two books originally published in 1916 and 1936.).

[16] Gerhold, S. On some non-holonomic sequences. Electronic Journal of Combinatorics 11, R87 (2004), 1-7. 
[17] Gerhold, S. Combinatorial Sequences: Non-Holonomicity and Inequalities. PhD thesis, J. Kepler University Linz, 2005.

[18] Gessel, I. M. Symmetric functions and P-recursiveness. Journal of Combinatorial Theory, Series A 53 (1990), 257-285.

[19] Hardy, G. H. Ramanujan: Twelve Lectures on Subjects Suggested by his Life and Work, third ed. Chelsea Publishing Company, 1978. Reprinted and Corrected from the First Edition, Cambridge, 1940.

[20] Henrici, P. Applied and Computational Complex Analysis, vol. 2. John Wiley, 1974.

[21] Jordan, C. Calculus of Finite Differences. Chelsea Publishing Company, 1965. First Edition, Budapest, 1939.

[22] Klazar, M. Non P-recursiveness of numbers of matchings or linear chord diagrams with many crossings. Advances in Applied Mathematics 30 (2003), 126-136.

[23] Klazar, M. Non-holonomicity of the sequence $\log 1, \log 2, \log 3, \cdots$. Preprint, Feb. 2005. Available from ArXiv:math.C0/0502141.

[24] LindelöF, E. Le calcul des résidus et ses applications à la théorie des fonctions. Collection de monographies sur la théorie des fonctions, publiée sous la direction de M. Émile Borel. Gauthier-Villars, Paris, 1905. Reprinted by Gabay, Paris, 1989.

[25] Lipshitz, L. The diagonal of a $D$-finite power series is D-finite. Journal of Algebra 113 (1988), 373-378.

[26] Lipshitz, L. D-finite power series. Journal of Algebra 122 (1989), 353-373.

[27] Madsen, R. W. Generalized binomial distributions. Communications in Statistics - Theory and Methods 22, 11 (1993), 3065-3086.

[28] Mainardi, F., And Gorenflo, R. On Mittag-Leffler-type functions in fractional evolution processes. Journal of Computational and Applied Mathematics 118, 1-2 (2000), 283-299.

[29] Milne-Thomson, L. M. The Calculus of Finite Differences. Chelsea Publishing Company, 1981. Reprinted from the original edition, London, 1933.

[30] Odlyzko, A. M. Asymptotic enumeration methods. In Handbook of Combinatorics, R. Graham, M. Grötschel, and L. Lovász, Eds., vol. II. Elsevier, 1995, pp. 1063-1229.

[31] Oppenheim, A. On an arithmetic function. Journal of the London Mathematical Society 1 (1926), 205-211.

[32] Pickard, W. F. On polylogarithms. Publicationes Mathematicae Debrecen, 15 (1968), 33-43.

[33] Rubel, L. A. A survey of transcendentally transcendental functions. American Mathematical Monthly 96, 9 (1989), 777-788.

[34] Slater, L. J. Generalized Hypergeometric Functions. Cambridge University Press, 1966. 
[35] Stanley, R. P. Differentiably finite power series. European Journal of Combinatorics 1 (1980), 175-188.

[36] Szekeres, G., And Turán, P. Über das zweite Hauptproblem der "Factorisatio Numerorum". Acta Litt. Sci. Szeged 6 (1933), 143-154.

[37] Teufl, E. On the asymptotic behaviour of analytic solutions of linear iterative functional equations. Aequationes Math. 73, 1-2 (2007), 18-55.

[38] Valiron, G. Sur le calcul approché de certaines fonctions entières. Bulletin de la Société Mathématique de France 42 (1914), 252-264.

[39] Wasow, W. Asymptotic Expansions for Ordinary Differential Equations. Dover, 1987. A reprint of the John Wiley edition, 1965.

[40] Whittaker, E. T., And Watson, G. N. A Course of Modern Analysis, fourth ed. Cambridge University Press, 1927. Reprinted 1973.

[41] Wright, E. M. The asymptotic expansion of integral functions defined by Taylor series. Philosophical Transactions of the Royal Society of London. Series A, Mathematical and Physical Sciences 238, 795 (1940), 423-451.

[42] Wright, E. M. The generalized Bessel function of order greater than one. Quarterly Journal of Mathematics 11 (1940), 36-48.

[43] Wright, E. M. The asymptotic expansion of integral functions defined by Taylor series (second paper). Philosophical Transactions of the Royal Society of London. Series A, Mathematical and Physical Sciences 239, 804 (1941), 217-232.

[44] Zeilberger, D. A holonomic systems approach to special functions identities. Journal of Computational and Applied Mathematics 32 (1990), 321-368. 